\title{
Increased expression of jagged-1, Notch-1 and Notch-4 in nasal mucosa of patients with allergic rhinitis
}

\author{
Eric Hjalmarsson ${ }^{1}$, Marianne Petro ${ }^{1}$, Susanna Kumlien Georén ${ }^{2}$, Ola Winquist ${ }^{3}$, and Lars \\ Olaf Cardell ${ }^{4}$
}

${ }^{1}$ Karolinska Institutet

${ }^{2}$ Karolinska Insitutet

${ }^{3}$ ABClabs

${ }^{4}$ Department of Otorhinolaryngology

February 12, 2021

\section{Dear Editor,}

$\mathrm{CD}^{+} \mathrm{T}$ helper 2 (Th2) cells play a key role in progression of allergic rhinitis (AR). Traditionally T-cells acquire the characteristics of Th2 cells in the presence of IL-4, a process that may be influenced by engagement of co-stimulatory factors expressed by antigen-presenting cells ${ }^{1}$. Notch is a co-stimulatory pathway for CD $4^{+}$ T-cells, and the importance of Notch for generation of Th2 cells and the development of allergic rhinitis has been suggested in animal studies ${ }^{2,3}$. However, its potential role in the advancement of allergy in humans remains unknown. Our group has previously demonstrated that neutrophils and nasal epithelial cells promote allergic mucosal inflammation by interacting with T-cells. Based on these findings, we hypothesized that neutrophils and epithelial cells in allergic nasal mucosa induce Notch signaling in CD4 ${ }^{+}$T-cells, promoting allergic inflammation. The present study analyzed Notch ligand expression on primary nasal epithelial cells and neutrophils along with Notch receptor expression on CD4 ${ }^{+}$T-cells in patients with pollen-induced AR off season and compared with healthy control (HC). In the appendix of this article information regarding patient characteristics and methods is supplemented.

Expression of Notch1-4 on T-cells was assessed by FACS. The fraction of CD4 ${ }^{+}$T-cells expressing Notch1,4 in mucosa was significantly higher in AR compared to HC (Fig 1B). No differences in Notch expression were detected in blood derived CD4 ${ }^{+}$T-cells (Fig 1A). In animal models of allergic rhinitis, JAG1-Notch1,4 signaling has been demonstrated to polarize T-cell responses towards Th2, promoting an allergic inflammation ${ }^{3,4}$. Against the backdrop of our findings in CD4 ${ }^{+}$T-cells, it is tempting to suggest that the increase in Notch1,4 in allergic mucosa might be a sign of increased Th2 cell polarization and that Notch signaling maintain TH2 inflammation and consequently AR progression. In the present study no difference in the expression of Notch was detected on $\mathrm{CD} 4^{+} \mathrm{T}$-cells in peripheral blood. However, this study was performed outside the pollen season, and it cannot be excluded that a similar signal can also be seen in the blood in patients with pollen induced symptoms. Further the expression of JAG1, JAG-2, DLL-1, and DLL-4 was also assessed with FACS. The fraction of epithelial cells expressing JAG-1 and DLL-1 was significantly elevated in AR compared to HC. (Fig 2A). The increased expression of JAG-1 and DLL-1 among patients with AR could be further corroborated by analyzing median fluorescence intensity (MFI) (Fig 2B,C). Although significant, the expression levels of DLL-1 on the surface of epithelial cells were notably lower than the corresponding expression levels of JAG-1. (Fig 2B,C). There are conflicting theories regarding in what way Notch signaling regulates T-cell functions. However, there are evidence for an instructive model where JAG-Notch1,2 interaction initiates Th2 cell differentiation, and DLL-Notch3 promotes T helper 1 (Th1) cell differentiation ${ }^{5}$. Our result illustrates a JAG-1>>DLL signaling gradient by epithelial cells supporting a Th2 outcome (Fig 
2B,C). Further, the fraction of neutrophils expressing JAG-1 was significantly increased in AR compared to $\mathrm{HC}$, in samples derived from mucosa and blood (Fig 2D,G). In addition, the expression levels of JAG-1 as measured by MFI, were significantly increased on neutrophils from AR compared to neutrophil from HC, in samples derived from mucosa and blood (Fig 2E,F and H,I).

Together with our previous report of antigen-presenting epithelial cells, our data suggest that the increased expression of JAG-1 in epithelial cells in allergic mucosa induce a sustained Th2 polarization of Notch1 and Notch 4 expressing T-cells. In analogy, neutrophils have also been shown to present antigens to T-cells ${ }^{6}$. Hence, it might be that neutrophils in AR mucosa present antigen to T-cells promoting a Th2 polarization in $\mathrm{CD} 4^{+}$T-cells by increased expression of JAG- 1 .

Wk 600

Increased expression of jagged-1, Notch-1 and Notch-4 in nasal mucosa of patients with allergic rhinitis

Eric Hjalmarsson ${ }^{1}$, Marianne Petro ${ }^{1}$, Susanna Kumlien Georén ${ }^{1}$, Ola Winqvist ${ }^{2}$, Lars Olaf Cardell ${ }^{1,3}$

1. Division of ENT Diseases, Department of Clinical Science, Intervention and Technology, Karolinska Institutet, Stockholm, Sweden

2. ABClabs, Biomedicum, Stockholm Sweden

3. Department of Otorhinolaryngology, Karolinska University Hospital, Stockholm, Sweden

Corresponding author:

Professor Lars Olaf Cardell

Division of ENT Diseases, Department of Clinical Science Intervention and Technology, Karolinska Insitutet, Stockholm, Sweden

Phone: $+46(0) 858581453$

E-mail: lars-olaf.cardell@ki.se

\section{Acknowledgement of funding}

VR, ALF

\section{Conflict of Interest}

The authors declare no conflict of interest.

\section{Authorship contribution}

Eric HJALMARSSON and Marianne PETRO have made substantial contributions in acquisition and data analysis. Eric HJALMARSSON have been involved in drafting the manuscript. All authors have been involved in conception, design, data interpretation and manuscript revising. All authors have read and approved the final manuscript.

Figure 1, Notch1-4 on T-cells in nasal mucosa and blood .Fraction of CD4 T-cells expressing Notch in A: peripheral blood and B: nasal mucosa. C: Flow cytometry gating strategy for detection of Notch expression. Patients with allergic rhinitis (AR), $(n=9)$, healthy controls $(H C),(n=11)$. Horizontal lines represent the mean value and SEM. $\mathrm{p}<0.01=* *$.

Figure 2, Expression of Notch ligands on epithelial cells and neutrophils A: Fraction of epithelial cells expressing Notch ligands. Median fluorescence intensity of B: JAG-1 and C: DLL-1. D: Fraction of neutrophils in nasal mucosa expressing Notch ligands. Median fluorescence intensity of E: JAG-1 and F: DLL-1. G: Fraction of neutrophils in blood expressing Notch ligands. H: Median fluorescence intensity of I: 
JAG-1 and J: DLL-1. Patients with allergic rhinitis (AR), $(\mathrm{n}=9)$, healthy controls $(\mathrm{HC}),(\mathrm{n}=11)$. Horizontal lines represent the mean value and SEM. $\mathrm{p}<0.05=^{*}, \mathrm{p}<0.01=* *$.

1. Coquet JM, Rausch L, Borst J. The importance of co-stimulation in the orchestration of $\mathrm{T}$ helper cell differentiation. Immunol Cell Biol. 2015;93(9):780-788.

2. Shi L, Ma Y, Zheng C, Zhang Q. The effect of blocking Notch signaling by gamma-secretase inhibitor on allergic rhinitis. Int J Pediatr Otorhinolaryngol. 2017;98:32-38.

3. Tindemans I, Lukkes M, de Bruijn MJW, et al. Notch signaling in T cells is essential for allergic airway inflammation, but expression of the Notch ligands Jagged 1 and Jagged 2 on dendritic cells is dispensable. J Allergy Clin Immunol. 2017;140(4):1079-1089.

4. Xia M, Harb H, Saffari A, Sioutas C, Chatila TA. A Jagged 1-Notch 4 molecular switch mediates airway inflammation induced by ultrafine particles. J Allergy Clin Immunol. 2018;142(4):1243-1256 e1217.

5. Tindemans I, Peeters MJW, Hendriks RW. Notch Signaling in T Helper Cell Subsets: Instructor or Unbiased Amplifier? Front Immunol.2017;8:419.

6. Polak D, Hafner C, Briza P, et al. A novel role for neutrophils in IgE-mediated allergy: Evidence for antigen presentation in late-phase reactions. J Allergy Clin Immunol. 2019;143(3):1143-1152 e1144.
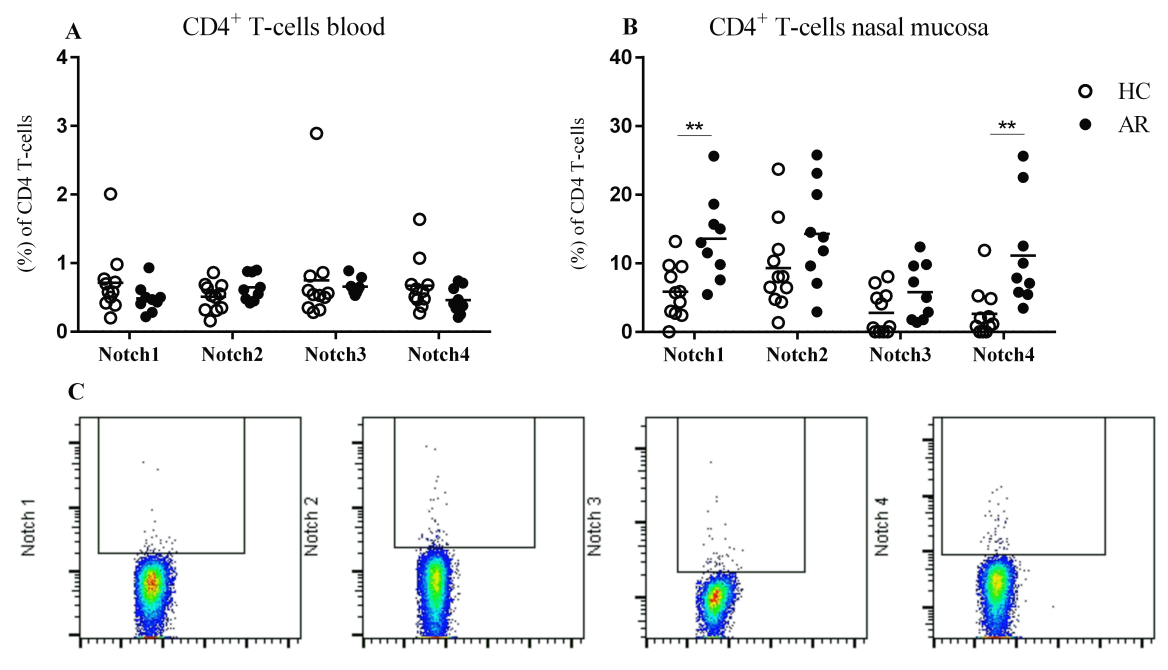

FSC-A

FSC-A

FSC-A

FSC-A 
Epithelial cells in nasal mucosa
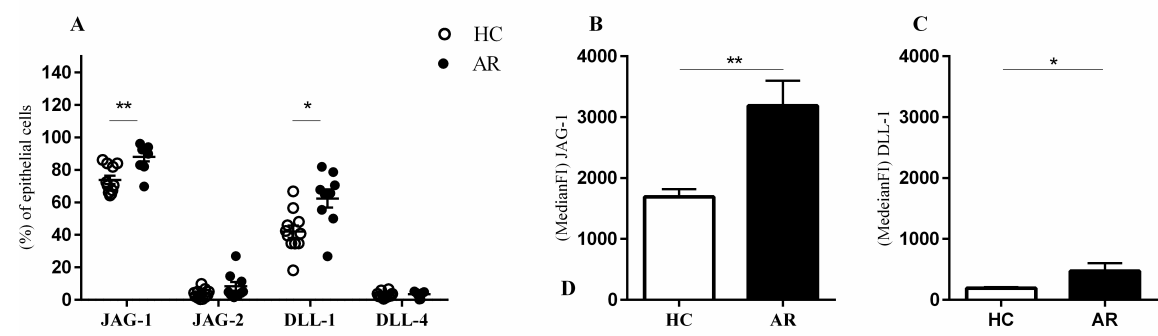

Neutrophils in nasal mucosa
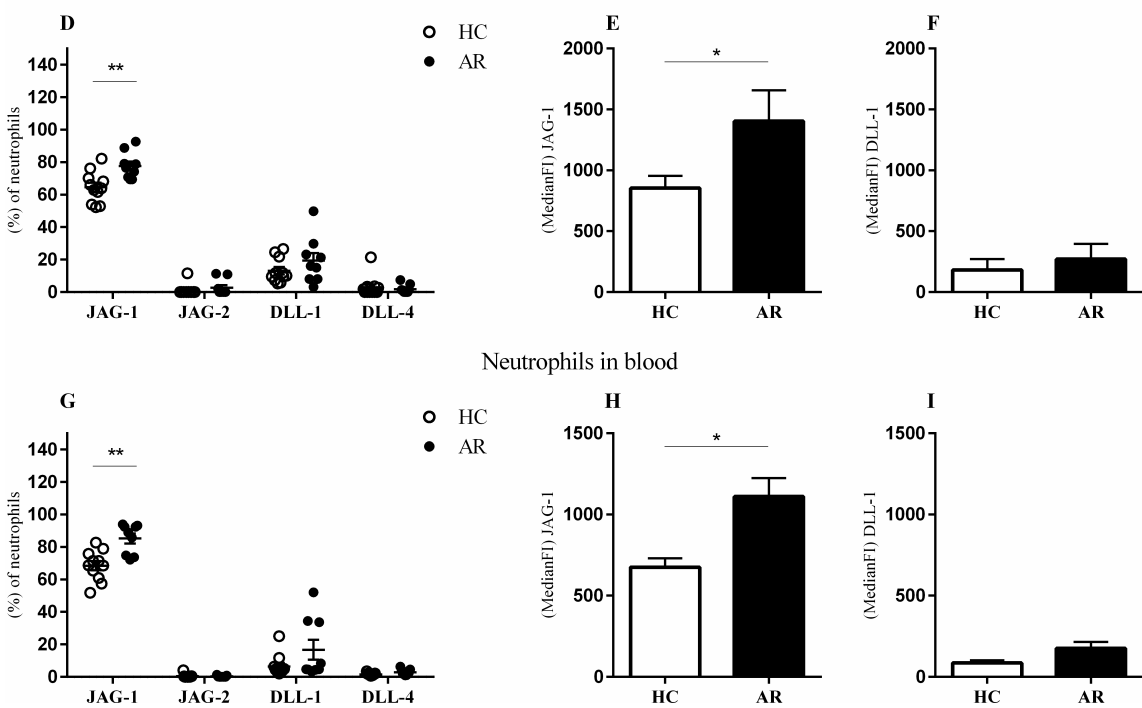

Neutrophils in blood
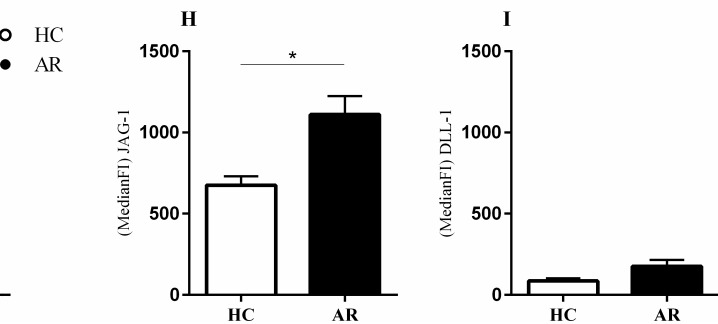